\title{
On supersonic convection in stellar atmospheres
}

\author{
D. R. Xiong ${ }^{1}$ and L. Deng ${ }^{2}$ \\ ${ }^{1}$ Purple Mountain Observatory, Chinese Academy of Sciences,Nanjing 210008, \\ email:xiongdr@pmo.ac.cn \\ ${ }^{2}$ National Astronomical Observatories, Chinese Academy of Sciences, Beijing 100012, \\ email:licai@bao.ac.cn
}

\begin{abstract}
It follows from the local mixing length theory that the convection in the atmospheres of yellow and red giants and super-giants become supersonic. In this work we studied the physical reality of such phenomenon and its possible consequences on the structure and evolution of stars involving such situations. The main conclusion is that the supersonic nature of convection as predicted by the local mixing length theory has been overestimated. If supersonic convection is not an artifact in all situations, it is the case at least for all yellow giants and super-giants. We believe that such an artifact is due to the imperfect of the mixing length theory. Owing to the fact that convective energy transport in the atmospheres of yellow giants and super-giants is quite negligible, these artifacts have very limited consequences on the structure and evolution of these stars. However, it is not the case for red giants and super-giants whose properties can be seriously affected by this overestimation. Our investigation shows that the theoretical red phases of stars under consideration are somewhat too blue as predicted with the usual mixing length theory. To this aim, full hydrodynamic simulation is needed in order to clear the doubts on the existence of supersonic convection in these red objects.
\end{abstract}

Keywords. Convection, stars: evolution, stars: interiors, stars: late-type

\section{Introduction}

Although being non-satisfactory in many aspects, the mixing length theory is still the most applied treatment of convection in stellar physics. The reasons for such a situation can be understood in the following way:

- It is straightforward and therefore is easy to be accepted;

- It is rather simple therefore can be easily adapted into applications;

- If our concerns are not focused on non-local convection phenomena such as convective overshooting, the local mixing length theory is still a fairly good primary approximation. This is mainly due to the fact that convective energy transport is highly effective in deep interiors of stars, so that the temperature gradient are very close to its adiabatic one, and this is independent of the convection theory used. In this case, the whole extent of convective region is fixed only by the structure of the so called super-adiabatic convective zone, any desired depth of convective zone can always be secured by adjustment of the mixing length.

It is found out long time ago that when applying mixing length theory for the calculations of models for red- and yellow-giants and super-giants, the regions atop of their surface convective zone will become supersonic. Problems related with the reality of supersonic convection, and its possible effects on the structure of stars have never drawn enough attention in the community, and therefore have not been studied thoroughly. Deng \& Xiong (2001) questioned the physical existence of supersonic convection. It is 
pointed out in that work that the local mixing length theory is not applicable when convection becomes supersonic for it overestimates supersonic property. Deng \& Xiong (2001) gave a modification to the local mixing length theory as a compromise, using which the effects of supersonic convection on the evolution of red giants and super-giants is investigated.

Following a non-local convection theory, we have carried careful analysis of cases of supersonic convection revealed by the local mixing length theory, the theoretical arguments and the main results are presented in the following.

\section{Supersonic convection in atmosphere of red and yellow giants and supergiants: the local mixing length theory}

Cai (2006) calculated the structure of convective envelopes for $1-60 \mathrm{M}_{\odot}$ stars at various evolutionary stages using the local mixing length theory, the results defined regions in the HR diagram where supersonic convection happens in corresponding stellar models. Fig. 1 shows a 3 dimensional distribution of supersonic convection as predicted by the local mixing length theory, where the solid dots are the models with subsonic convection, the circles are the models with supersonic convection, and the size of the symbols is proportional to the maximum Mach number of convective motion $M=\max \left\{u^{\prime} / C_{s}\right\}$, where $u^{\prime}$ is the root mean square velocity of turbulent convective motion, $C_{s}=\left(\Gamma_{1} P / \rho\right)^{1 / 2}$ is the local adiabatic sound speed. It is clear from Fig. 1 that convection in all models with $M<2 M_{\odot}$ is subsonic. Starting from $M \sim 2 M_{\odot}$, the yellow giant phases become supersonic. Going to higher luminosity (higher stellar mass), supersonic convection becomes stronger and extends towards lower effective temperatures. In the HR diagram, the supersonic convection models define a top-down wedge like strip inclining towards upper-right direction. For low and intermediate luminosities, supersonic convection happens in yellow giant stage, while for high luminosity giants and super-giants, supersonic convection stretches into the red phase near the Hayashi limits. The Mach number of supersonic convection tends to grow with higher luminosities. The extreme case happens in yellow color, supersonic convection weakens towards the redder colors.

It worth emphasizing that the distribution of supersonic convection models in the HR diagram shown in Fig. 1 is a result of the local mixing length theory, therefore might not be physically real. We are going to explain in the following the meaning of Fig. 1 based on the formula of mixing length theory. The expression of convective speed in the scope of mixing length theory is (Böhm-Vitense 1958),

$$
u_{r}^{\prime 2}=\frac{1}{4} \alpha^{2} Q \frac{R T}{\bar{\mu}} \frac{\gamma}{(1+\gamma)}\left(\nabla-\nabla_{a d}\right)=\frac{\alpha^{2} Q}{4 \Gamma_{1}} C_{s}^{2} \frac{\gamma}{(1+\gamma)}\left(\nabla-\nabla_{a d}\right),
$$

where $\alpha=l / H_{P}$ is the mixing length parameter, $Q=-\left(\frac{\partial \ln \rho}{\partial \ln T}\right)_{P}$ the gas expansion coefficient, $C_{s}$ the local sound speed, $\Gamma_{1}$ the adiabatic index, $\gamma$ the effective Peclet number of turbulent convection. $Q$ and $\Gamma_{1}$ are slow varying functions of temperature and density of order unity. Hence, the Mach number of convective motion depends primarily on $\left(\nabla-\nabla_{a d}\right) \gamma /(1+\gamma)$. When $\gamma \gg 1$, we have $\nabla \sim \nabla_{a d}+\frac{4}{9 \gamma}\left(\nabla_{r}-\nabla_{a d}\right) \sim \nabla_{a d}$, where $\nabla_{r}$ is the radiative temperature gradient, therefore convection will be subsonic in this case. When $\gamma \ll 1, \nabla=\nabla_{r}-\frac{9}{4} \gamma^{2}\left(\nabla_{r}-\nabla_{a d}\right) \sim \nabla_{r}$, and convection may become supersonic. Therefore two conditions must be realized at the same time to turn convection into supersonic: 1 ). $\nabla_{r} \gg 1$, and 2 ). $\gamma \lesssim 1$.

It is well known that the radiative opacity reaches a maximum near $10^{4} \mathrm{~K}$ due to the overlay of hydrogen absorption peak on the maximum of the Plankian distribution of 


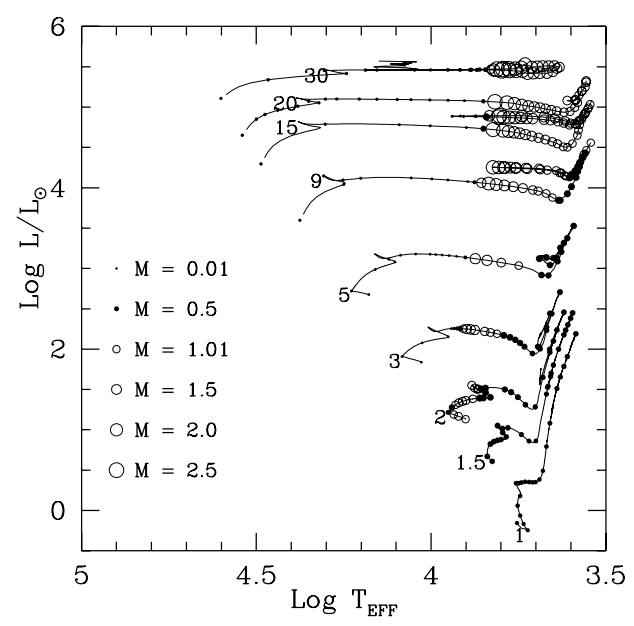

Figure 1. A distribution of models with/without supersonic convection in the HR diagram using the local mixing length theory. The solid lines are the evolutionary tracks with initial stellar mass labeled. The solid dots are for subsonic convection model, the open circles are for models with supersonic convection. The size of the symbols is proportional to the maximum Mach number found in that model, as indicated in the inlet.

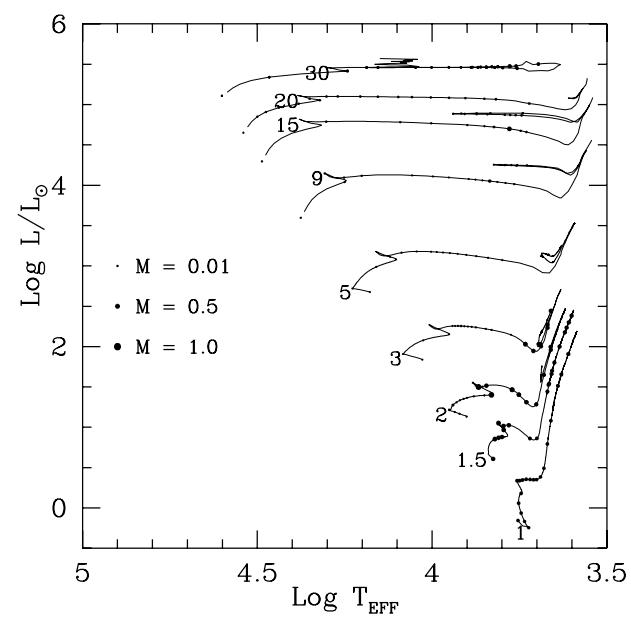

Figure 2. The same as Fig. 1, but calculated using our non-local statistical theory of convection. The convective parameter for both Fig. 1 and this figure are calibrated by the known structure of solar convective zone.

radiation at this temperature. This temperature is just located in the hydrogen ionization zone. For low luminosity dwarfs, density of the hydrogen ionization zone is high enough, and convection is very effective, so that $\gamma \gg 1$. Taking the Sun as an example, except in a very shallow super-adiabatic convective zone near the top of the convective envelope, the temperature gradient is very close to its adiabatic value $\nabla_{a d}$. Based on these arguments, convective motion in dwarfs and low luminosity giants is subsonic, i.e. $M=u^{\prime} / C_{s}<1$. For increasing stellar luminosity, the density in the hydrogen ionization zone decreases, convective energy transport becomes less and less effective, so that $\gamma<1$. The temperature gradient approaches the radiative one, therefore the Mach number of convective motion grows for increasing luminosity, and eventually enters supersonic regime. 
Why does the strongest supersonic convection happen in the specific temperature range of yellow giants and super-giants? Answer to this question is the following: for given luminosity and mass, the radiative temperature gradient $\nabla_{r}$ in the hydrogen ionization zone grows when stellar effective temperature drops down, and convection tends to be supersonic; On the other hand, however, for lower effective temperatures, the hydrogen ionization zone sinks deeper into into the atmospheres, therefore the density and $\gamma$ increase, and this leads to smaller super-adiabatic gradient $\nabla-\nabla_{a d}$. The interplay of these two factors bring about a maximum supersonic property for the convective zone at a certain effective temperature. Going away from this critical point on both directions, supersonic convection weakens. This is a straightforward explanation of the distribution of supersonic convection models in Fig. 1.

\section{Is supersonic convection physically real?}

We need to clarify again that the local mixing length theory is not suitable when convection becomes supersonic, because an implicit hypothesis of $\alpha\left(\nabla-\nabla_{a d}\right) \gamma /(1+\gamma) \ll 1$ is made in establish the mixing length formulism (Deng \& Xiong 2001). Direct application of the theory valid only under such assumption to conditions with $\alpha\left(\nabla-\nabla_{a d}\right) \gamma /(1+\gamma) \geqslant$ 1 will merely alters the numerical value of the Mach number of convective motion, not causes the model to collapse. We will address this point latter. An other implicit hypothesis made in the scope of the local mixing length theory has consequences far more serious, which is about the existence of supersonic convection. In the derivation of the formulae of the local mixing length theory, one has to assume that the super-adiabatic temperature gradient $\nabla-\nabla_{a d}$ is constant within the mixing length scale. In reality, however, $\nabla-\nabla_{a d}$ is not only a variable, but also a fast changing variable at the mixing length scale level. Spiegel (1963) proposed a generalized mixing length theory just to attach such problem, in that he suggested using a certain kind of mean value of $\nabla-\nabla_{a d}$ within a mixing length to substitute the local value used in the original theory. Such a modification actually made Speigel's theory the first non-local mixing length theory of stellar convection.

Making use of our non-local statistical theory of convection, we have done calculation for the same envelope models along evolutionary phases as indicated in Fig. 1. The convective parameters as chosen as such: $c_{1}=0.87, c_{2}=0.437$, this choice makes the depth of solar convective zone the same as that deduced from the local theory, $r_{c} / r_{\odot} \sim$ 0.713 , where $r_{c}$ is the radius at the bottom of convective zone. The maximum Mach number of turbulent convection for all the models are presented in Fig 2 with solid dots, again the size is proportional to value of Mach number of the model. For models lying on the red giant and super-giant phases with $M=5-30 M_{\odot}$, we could not make the final results under non-local theory due to numerical instabilities encountered during our calculations. It follows from Fig. 2 that convection is always subsonic for all models having results of non-local calculations. The Mach numbers of convective motions in this case are subsonic and far smaller than what are expected from the local mixing length theory!

Fig. 3 shows for a $M=20 M_{\odot}$ yellow super-giant model the relative fluctuations of turbulent velocity and temperature, and fractional convective flux as functions of depth in $\ln P$, where the solid lines are for the non-local convection models, while the dashed and dotted lines are those of local expression. The star considered here has a luminosity of $\log L / L_{\odot}=5.0441$, and an effective temperature of $\log T_{e}=3.7617$. It is clear from Fig. 3 that turbulent velocity, temperature fluctuation and fractional convective flux of 


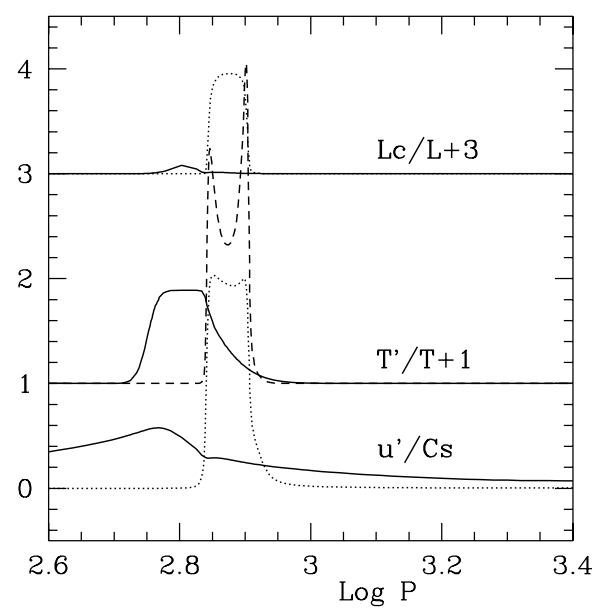

Figure 3. The internal structure of a $20 M_{\odot}$ star with $\log L / L_{\odot}=5.0441, \log T_{e}=3.7617$. Calculations are made using the local mixing length theory (dotted and dashed lines)and our non-local theory (solid line). Showing here are fluctuations of turbulent velocity $\left(u^{\prime} / C_{s}\right)$ and temperature $\left(T^{\prime} / \bar{T}\right)$, and the fractional convective flux $\left(L_{c} / L\right)$ as functions of depth in $\log P$.
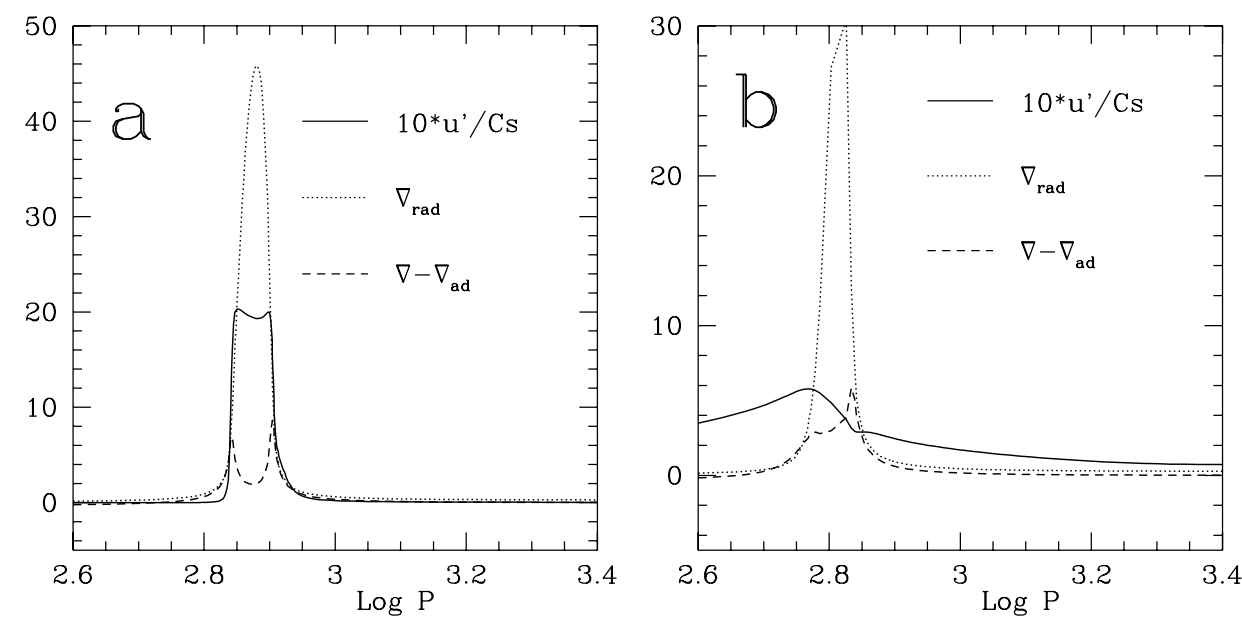

Figure 4. Radiative temperature gradient $\nabla_{r a d}$, super-adiabatic temperature gradient $\nabla-\nabla_{a d}$ and turbulent Mach number $u^{\prime} / C_{s}$ as functions of depth for the same stellar model as in Fig. 3. a). local convection model; b). non-local convection model.

non-local models are all much smaller than those predicted by the local theory. Why is it so? Following our investigations, there are two reasons:

Firstly, as we expressed before, when using the local mixing length theory, the superadiabatic temperature gradient $\nabla-\nabla_{a d}$ is assumed to be its local value. In fact, $\nabla-\nabla_{a d}$ varies greatly in the scale of the mixing length. Figs. $4 \mathrm{a}$ and $4 \mathrm{~b}$ show the radiative temperature gradient $\nabla_{r a d}$, super-adiabatic temperature gradient $\nabla-\nabla_{a d}$ and the Mach number of turbulent motions as functions of depth in the cass of local and non-local treatments respectively. From Fig. 4a, one can see that $\nabla_{\text {rad }}$ and $\nabla-\nabla_{a d}$ are greater than one only in a very narrow range of depth $\left(\sim 0.23 H_{P}\right)$, being much smaller than the local mixing length. Therefore the local mixing length theory overestimates turbulent velocity, temperature fluctuation and convective flux. This is exactly the reason for 
Spiegel to raise his generalized mixing length theory. The non-local effects of $\nabla-\nabla_{a d}$ has also been addressed in our non-local convection theory. This is also one of the reasons why convection in yellow giants and super-giants is not supersonic as shown in Fig. 2. Considering the effects of boundary of convection zone on the mixing length, it has been modified by Hoffmeister \& Weigert (1964) and Böhm \& Stükle (1967) that $l=\min \left\{\alpha H_{P}, Z\right\}$, where $\mathrm{Z}$ is the distance to the nearest boundary of convection zone, or simply $l=Z$ (Canuto \& Mazetelli 1991), so that the supersonic convection can be mostly superseded.

Secondly, regions with strong turbulence bound to diffuse into areas with weaker turbulence owing to the non-local properties of turbulent convection. The results derived from our non-local convection theory (Xiong 1989) are shown in Fig. 5, where the solid line is the work done by buoyant force to unit mass $W_{b}=Q g V, Q=-(\partial \ln \rho / \partial \ln T)_{P}$ is the gas expansion coefficient, $g=G M_{r} / r^{2}$ is the local gravitational acceleration, and $V=\overline{u_{r}^{\prime} T^{\prime}} / \bar{T}$ is the correlation between (radial) turbulent velocity and temperature. The dot-dashed line denotes the energy lost per unit mass due to turbulent diffusion $d L_{t} / d M_{r}$, where $L_{t}=2 \pi r^{2} \bar{\rho} \overline{u_{r}^{\prime} w_{i}^{\prime} w^{\prime i}}$ is the turbulent energy penetrating the sphere of radius $r$. Both quantities are measured in unit of turbulent (molecule) viscous dissipation rate $\epsilon_{2}=\overline{\sigma^{i k}\left(u^{\prime}\right) \nabla_{k} u_{i}^{\prime} / \bar{\rho}}$, where $\sigma^{i k}\left(u^{\prime}\right)$ is the viscous stress tensor. It follows from Fig. 5 that, within convectively unstable zone $(V>0)$, the buoyant work $W_{b}>0$, but the turbulent diffusion is negative $d L_{t} / d M_{r}<0$. That is to say, turbulence gains power due to work done by buoyant force, while losses energy due to turbulent diffusion and molecular viscosity. In the case of steady convection, the three parties reach an equilibrium, $W_{b}+d L_{t} / d M_{r}-\epsilon_{2}=0$. In the overshooting zone $(V<0)$, we have $W_{b}<0$ and $d L_{t} / d M_{r}>0$, i.e. buoyant force does negative work to therefore breaks down turbulent eddies, while viscous force is always a dissipation. In order to keep turbulence steady, some energy source outside the overshooting zone must exist, and that is nothing but the turbulent kinetic energy flux. This physical picture is clear enough. Although the amplitude of turbulent diffusion $d L_{t} / d M_{r}$ shown in Fig. 5 depends on the the non-local theory used, the existence of turbulent kinetic energy flow from convectively unstable zone to the attached overshooting zone is fatal for overshooting to survive. Therefore, by taking into account the non-local effects of convection, the convective motion within the unstable zone must be weakened, in other words, the local mixing length theory must have overestimated the strength of convective motion in the unstable zone near the boundary.

We would like to mention here that Spiegel's non-local mixing length theory considered only the first aspect of the non-locality. The second one, i.e. the effect of turbulent diffusion, was not taken into account in his work. Therefore, his theory still somewhat overestimates the efficiency of convective energy transport, and that is the reason for the mismatch between the observations on solar limb darkening and line profiles and the prediction make by that theory (Travis \& Matsushima 1973). Because of the overestimation of convective energy transport, the temperature gradient within solar atmosphere is made too small in the model. Furthermore, Spiegel's theory is also unable to explain the anti-correlation between turbulent velocity and temperature as observed in the outer layers of solar atmosphere. In contrast, our non-local statistical theory of convection behaves perfect in these two observational tests (Xiong \& Cheng 1992). In addition, our model for solar convective zone can fit very well the depth that is secured by helioseismology, and at the same time can correctly predict Lithium abundance in solar atmosphere (Xiong \& Deng 2002).

Taking into account the two factors discussed above, it is easy to understand why the local mixing length theory overestimates the strength of turbulent convection in the 


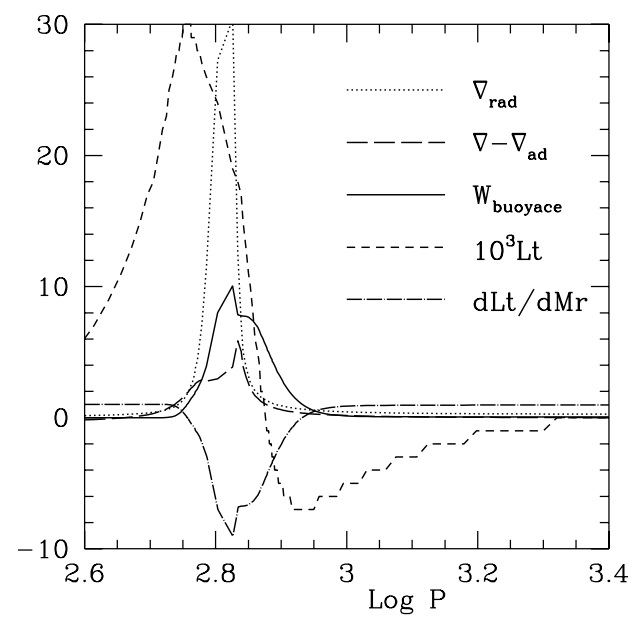

Figure 5. Work of buoyancy (the solid line), flux of turbulent kinetic energy $L_{t}$ (the dashed line), turbulent diffusion loss $d L_{t} / d M_{r}$ (the dot-dashed line), radiative temperature gradient $\nabla_{\text {rad }}$ (the dotted line) and the super-adiabatic temperature gradient (the long-dashed line) versus the depth for the same yellow super-giant as in Figs. $3 \& 4$.

unstable zone. Turbulence must become weaker if one fully incorporates the non-localities of turbulent convection (comparing Figs. 1 and 2, and Figs 4a and 4b).

\section{Conclusion and discussions}

Our analysis shows that the local mixing length theory indeed overestimates the strength of convective motions in the super-adiabatic convection zone. Considering the non-localities of turbulent convection, the strength must be a lot lower. Such a conclusion is independent of the flavor of non-local theory. Different theory alters the result quantitatively, not qualitatively. Our studies reveals that the supersonic convective phenomena as predicted for most (if not all) of the yellow giants and super-giants are artifacts, which can be understood by the defects in the local mixing length theory that overestimates convection in the super-adiabatic zone of stars. The shallower the outer convective zone, the worse the overestimation. The fake "supersonic convection" has limited consequences on the structure of yellow giants and super-giants, simply because the convective zone is very shallow, convective energy transport over there is highly ineffective, therefore the temperature gradient in that zone is almost radiative. However, for redder objects close to the Hayashi limit possessing more extended deep convective zones, convective energy transport is significant. The influence of this overestimation on the structure and evolution of red giants and super-giants becomes serious. Based on these arguments, we believe that the theoretical predictions of the red phases of stellar evolution near the Hayashi limit by the local mixing length theory (this is very general in evolutionary modeling) is somewhat too blue. This agrees with theoretical stellar evolution scenario and observations of the red populations in the HR diagram (Deng, Bressan \& Chiosi 1997, Deng \& Xiong 2001).

\section{Acknowledgements}

We would like to thank K.L. Chan for valuable discussions. This work is supported in part by NSFC through grants 10573022, 10273021 and 10333060. 


\section{References}

Böhm-VItense, E. 1958, Z. Astrophys. 46, 108

Böhm, K.H. \& Stükle, E., 1967, Z. Astrophys., 66, 487

Canuto, V.M. 1991, ApJ, 370, 295

Deng, L. Bressan, A. \& Chiosi, C., 1996, A $\& A$ 313, 145

Deng, L. \& Xiong, D.R., 2001, Chin. J. Astron. Astrophys. 1, 50

Hofmeister, E. \& Weigert, A., 1964, Z. Astrophys., 59, 119

Spiegel, E.A., 1963 ApJ 138, 216

Travis, L.D. \& Matsushima, S., 1973, ApJ 186, 975

Xiong, D.R., 1989, A\& A 209, 126

Xiong, D.R. \& Cheng, Q.L., 1992 A $\& A$ 254, 362

Xiong, D.R. \& Deng, L., 2002 MNRAS 336, 511

\section{Discussion}

RoxBURGH: The fact that you have not found supersonic convection does not mean you will not find it. If you go a different set of models, eg. large mass cool super-giants.

DenG: Definitely you are right. Actually we made the same point for our conclusion is drawn based only on the models having results from non-local theory, in which no supersonic convection was found. This does not include red giants and super-giants for which no results are available at present time.

Canuto: How did you represent non-locality?

DENG: Using statistical theory of correlations as of Xiong (1989).

KuPKA: How did you compute the non-local models? Did you evolve them with standard evolution code and made some relaxation taking the local model as a starting solution for say, a yellow super-giant?

Deng: We have taken the Padova evolutionary tracks. The non-local model was used only for the envelope computation. We are working on coupling the non-local model to an evolutionary code, but this is a difficult work.

WuCHTERL: I can confirm the absence of supersonic convection from the proto-stellar calculation of $\tilde{1} 00 \mathrm{~L}_{\odot}$, and $1 \mathrm{M}_{\odot}$-Hayashi line for the Kuhfuss/Wuchterl \& Feuchtinger (1998) model. What is the main difference between Wuchterl \& Feuchtinger (1998) and your non-local convection model?

DenG: As I said, our conclusion should not be qualitative different between different flavors of non-local theory, so I think it is natural to have similar results from your work. 